\title{
TRANSFERENCIA DE TECNOLOGÍA EN LA INDUSTRIA MEXICANA DEL CEMENTO
}

\author{
Roland Ziss \\ El Colegio de México
}

\section{INTRODUCCIÓN}

ESTE TRABAJO ESTUDIA LA transferencia de tecnología en la industria mexicana del cemento. El objetivo es el análisis cuantitativo y sectorial de la transferencia de tecnología, para ilustrar un fenómeno económico que es cada vez más importante en el marco de la división internacional del trabajo dentro del cual cristalizan los cambios estructurales de los países tanto industrializados como aquellos en vías de desarrollo.

La industria del cemento permite el estudio de la transferencia de tecnología desde varios puntos de vista. La homogeneidad y estandarización de su producto facilita comparaciones intrasectoriales. La producción de cemento no está sujeta a secretos patentados; la tecnología del proceso es conocida y la maquinaria y los equipos son de libre acceso en el mercado mundial. La industria mexicana del cemento es un sector industrial consolidado desde hace mucho tiempo; esto permite enfocar las características estructurales de la transferencia de tecnología dentro de un contexto histórico estable.

El método empleado en esta investigación se aproxima al proceso productivo situando sistemáticamente las observaciones microeconómicas, en el marco de la internacionalización de la producción. La información analizada, se obtuvo mediante una éncuesta realizada en las plantas cementeras. A través de cuestionarios estandarizados se obtuvieron datos de casos concretos de transferencia de tecnología, tanto en el pasado como en la actualidad, en 15 de las 30 plantas que actualmente están produciendo o se hallan en construcción. En la mayoría de los casos se contó con la cooperación del director técnico de la empresa o del jefe de producción; además se hicieron visitas extensivas a la planta y se profundizaron aspectos específicos con su personal. Otra fuente de información fueron las entrevistas a algunos de los proveedores de tecnología. En cuanto a la tecnología no incorporada, el Registro Nacional de Transferencia de Tecnología facilitó el acceso a los contratos sobre asistencia técnica. Además se consultó ma- 
terial informativo de varias instituciones relacionadas con el sector, como por ejemplo, la Cámara Nacional de la Construcción y la Cámara Nacional del Cemento.

Agradecemos profundamente la cooperación a todas las personas, empresas e instituciones que brindaron su ayuda, esperando que este trabajo sea útil para sus futuras actividades.

En este informe se resumirán de manera sucinta, los resultados sobresalientes de la investigación empírica. Se utilizará un concepto amplio de "tecnología": dado que se trata de la producción de un producto, se considerará como tecnología al conjunto de elementos físicos incorporados en maquinaria y equipo, y a los conocimientos para la producción de un producto. No será abordada la política empresarial ni la del Estado en materia tecnológica, ni las implicaciones de la transferencia de tecnología en la reformulación de la teoría económica de la internacionalización de la producción, ya que este proceso cada vez está más determinado por una fracción del capital en formación; el capital tecnológico. Se presentará, específicamente, un diagnóstico de las condiciones prevalecientes y del estado actual de la transferencia de tecnología en la industria mexicana del cemento.

En el capítulo 2 se hará un resumen del desarrollo y de la estructura de la industria mexicana del cemento. En el capítulo 3 se presentan algunas observaciones sobre el nivel tecnológico de este sector. En el capítulo 4 se presentarán los principales proveedores de la tecnología incorporada y no incorporada a los equipos y maquinarias. El capítulo 5 analiza las fases de un caso típico de transferencia de tecnología en la instalación de una nueva línea de producción, tomando en consideración a los agentes que intervienen y la capacidad de la empresa receptora. En el último capítulo se analizan los costos y la estructura de los mismos en dos casos concretos de transferencia de tecnología y se calcula el contenido de importación, utilizando los conceptos de costo y de peso del equipo.

\section{Desarrollo y estructura de la industria mexicana del CEMENTo}

Desde la fundación de las primeras plantas cementeras a principios de este siglo, la producción mexicana de cemento tuvo una expansión casi ininterrumpida debido a una demanda creciente y a una diferenciación de usos. Mientras que en los años 20 se usaba el cemento casi exclusivamente para manufacturar mosaicos para pisos de baño, cocina y pasillos, hoy en día el cemento es el material más importante en cualquier tipo de construcción, tanto por su función constructiva como por su proporción en los costos; además sirve como producto intermedio para una serie de elementos prefabricados.

La capacidad total instalada por año era en 1950 de 1.8 millones de toneladas, de 3.5 millones de toneladas en 1960 , de 8.0 millones de to- 
neladas en 1970, de 14.2 millones de toneladas en 1978 (según los datos de la Cámara Nacional del Cemento, en base a 300 días de producción por año) y se calcula que llegará a 32 millones de toneladas para fines de 1983 (según el Programa de Fomento para la Industria del Cemento, publicado en el Diario Oficial del 1o. de julio de 1980).

La demanda nacional se distribuye de dos formas: el uso directo para la ejecución de obras y el uso indirecto para la manufactura de elementos prefabricados, como bloques de concreto, piezas pretensadas, productos de asbesto cemento y mosaicos; se estima que aproximadamente dos tercios del cemento se dedica al uso directo y un tercio al uso indirecto. En cuanto a la distribución de la demanda nacional a nivel público y privado, se estima que cada uno de los sectores absorbe aproximadamente la mitad del cemento, si se incluyen los elementos prefabricados.

Las actividades de la construcción son sumamente sensibles a las coyunturas y muestran grandes variaciones. En particular, la demanda del sector público está sujeta a variaciones cíclicas de acuerdo con los períodos presidenciales. Como no existe una planificación integrada de la oferta y la demanda, en el pasado se presentaron períodos de escasez del cemento en el mercado que obligaron a importarlo de los países limítrofes. Causas importantes de los cuellos de botella de la oferta son: el largo plazo que requiere la ampliación de la capacidad instalada, los altos costos de inversión involucrados, la parte del financiamiento propio que se realiza mejor en época de auge y la rigidez del aparato productivo con un alto grado de automatización que impide una adaptación rápida a las coyunturas del mercado. En la gráfica 1 se representa esquemáticamente esta situación.

Actualmente casi todas las plantas cementeras están ampliando su capacidad instalada y muchas de ellas proyectan exportar cemento (sobre todo al sur de los Estados Unidos) cuando exista un exceso de producción. Se prevé que esta situación se concretará después del próximo cambio presidencial cuando las obras públicas estarán paradas por un cierto tiempo y la capacidad instalada habrá aumentado considerablemente; de 1979 a 1983 se espera una duplicación de la capacidad instalada de la producción cementera.

La influencia estatal en la producción cementera es mínima y se reduce a participaciones bancarias. Como el cemento es considerado un producto básico y de importancia estratégica para el desarrollo del país, medidas de fomento industrial están apoyando las inversiones privadas en la industria, por ejemplo, en forma de incentivos fiscales. Otro tipo de intervención del Estado se realiza a través del control de precios. Hasta hace poco tiempo el cemento tenía un precio controlado LAB fábrica; sin embargo no fue posible evitar la especulación en la distribución. Actualmente existe un decreto que garantiza un precio máximo del cemento en obra, según regiones, y se espera poder controlar mejor el mercado en tiempos de escasez. 


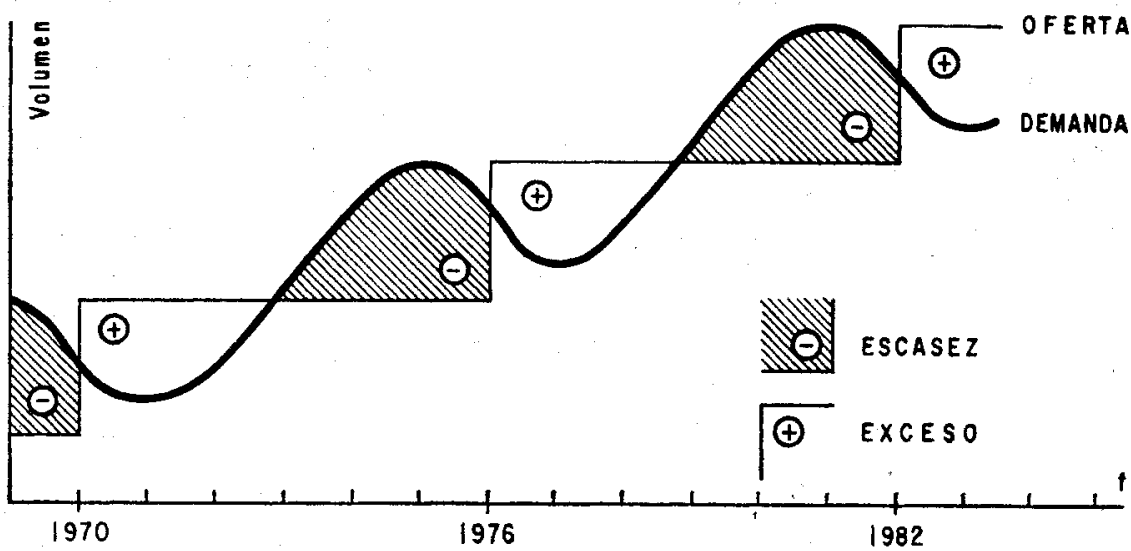

En 1978 tres grupos cementeros tenían el $71.3 \%$ de la capacidad total instalada: Cementos Mexicanos, con sede principal en Monterrey, 8 plantas y $25.7 \%$, Cementos Anáhuac, con sede principal en la Ciudad de México, 2 plantas y $23.0 \%$ y Cementos Tolteca, con sede principal en la Ciudad de México, 8 plantas y $22.6 \%$ (ver cuadro 1). Siguen en importancia tres grupos menores con 2 plantas cada uno: Cementos Apasco (11.8\%), Cementos Cruz Azul (9.6\%) y Cementos de Chihuahua (3.2\%). Los $4.1 \%$ restantes se reparten entre 4 fábricas independientes. La estructura actual de la industria mexicana del cemento es el resultado de un largo proceso de expansión y concentración que fue diferente para cada grupo. El grupo Anáhuac, por ejemplo, sufrió continuas ampliaciones en su planta del norte de la capital e inauguró, en 1968, una nueva en el Golfo de México. Los grupos Cementos Mexicanos y Tolteca no solamente ampliaron sus propias plantas, sino que se consolidaron también a través de la compra de plantas independientes.

La situación competitiva se ve afectada por las propias características del producto que dan lugar a una regionalización del mercado. La baja intensidad económica del cemento y los altos costos del transporte generan un mercado que se desarrolla alrededor de la fábrica. Dado que las 28 plantas existentes actualmente están bastante bien distribuidas en los estados y cerca de los centros de consumo, los productores, por lo general, cuentan con mercados protegidos. Únicamente las aglomeraciones más importantes del país y el exceso de producción son capaces de producir situaciones competitivas.

Casi todas las fábricas de cemento son sociedades anónimas; constituyen la excepción dos cooperativas que se forman por decreto presidencial en los años 30: Cementos Hidalgo en Nuevo León y Cementos Cruz Azul. 


\section{Cuadro 1}

Estructura de LA INDUSTRIA MEXICANA DEL CEMENTO

\begin{tabular}{|c|c|c|c|c|c|c|c|c|c|c|}
\hline \multirow{3}{*}{$\begin{array}{c}\text { Empresas } \\
\text { Cementeras }\end{array}$} & \multirow{3}{*}{$\begin{array}{l}\text { Participación } \\
\text { extranjera }\end{array}$} & \multicolumn{2}{|l|}{ T 1 po } & \multicolumn{3}{|c|}{1968} & \multirow{3}{*}{$\begin{array}{l}\mathrm{N}^{\circ} \mathrm{de} \\
\text { plantas }\end{array}$} & \multirow{2}{*}{\multicolumn{2}{|c|}{$\begin{array}{l}1978 \\
\text { Capacidad }\end{array}$}} & \multirow{3}{*}{$\begin{array}{l}\text { Instalada } \\
\text { Porciento }\end{array}$} \\
\hline & & Nacional & Cooperativa & $N^{0}$ de & Capacidad & instalada & & & & \\
\hline & & & & & $\begin{array}{l}\text { Toneladas } \\
\text { por dfa }\end{array}$ & Porciento & & $\begin{array}{l}\text { Tone] } \\
\text { por }\end{array}$ & $\begin{array}{l}\text { ladas } \\
\text { dIa }\end{array}$ & \\
\hline Mexicanos & & $\mathrm{x}$ & & 5 & 3730 & 15.5 & $8(1)$ & 12 & 055 & 22.5 \\
\hline Anáhuac & & $x$ & & 2 & 3550 & 14.8 & 2 & 10 & 900 & 23.0 \\
\hline Tolteca & $x$ & & & 7 & 6300 & 26.2 & 8 & 10 & 720 & 22.6 \\
\hline Apasco & $\mathrm{x}$ & & & 2 & 2750 & 11.4 & 2 & & 600 & 11.8 \\
\hline Cruz AzuI & & & $\mathrm{x}$ & 2 & 2750 & 11.4 & 2 & & 550 & 9.6 \\
\hline Chihuahua & & $x$ & & 1 & 900 & 3.7 & 2 & & 500 & 3.2 \\
\hline Resto (2) & & $\mathrm{x}$ & $x$ & $7(1)$ & 4040 & 16.8 & 4 & & 2040 & 4.3 \\
\hline Tota 1 & & & & 26 & 24020 & 99.8 & 28 & & 7365 & 100.0 \\
\hline
\end{tabular}

1 Inclusive las 3 plantas de Cementos Guadalajara que compraron Cementos Mexicanos entre 1974 y 1976.

2 De las pequeñas empresas cementeras independientes, una es cooperativa.

Fuente: Cámara Nacional del Cemento. 
Cuando los antiguos propietarios de Cruz Azul, de nacionalidad inglesa, anunciaron el cierre de la planta por falta de rentabilidad, se les expropió a cambio de una indemnización, luego las instalaciones fueron entregadas en propiedad cooperativa a los trabajadores que también organizaron la extracción de la materia prima y la red de distribución de la misma forma.

Dos grupos cementeros cuentan con un $49 \%$ de participación extranjera en el capital social: Cementos Tolteca, del grupo inglés Blue Circle, y Cementos Apasco del grupo suizo Holderbank. Ambos grupos extranjeros son empresas trasnacionales productoras de cemento, que participan en fábricas de muchos países. La mexicanización de la industria cementera, es decir, la reducción de la participación extranjera en grado minoritario, se llevó a cabo a principios de los años 70 .

\section{Nivel tecnológico de la industria mexicana del CEMENTo}

Para comprender el papel que juega la transferencia de tecnología en la industria mexicana del cemento partimos de un análisis del nivel tecnológico de la maquinaria usada actualmente, tomando en cuenta las innovaciones recientes en este sector. Según el flujo de materiales, desde la extracción de la materia prima hasta el envío del producto, podemos distinguir 6 departamentos principales:

- Trituración de la materia prima (caliza y arcilla).

- Homogeneización del material para obtener la composición química requerida.

- Molienda de crudo.

- Calcinación.

- Molienda de cemento.

- Envase.

A continuación se harán algunas observaciones relacionadas con los hornos de calcinación, que son el núcleo fundamental de la producción del cemento, y determinan la capacidad de una cementera. Los hornos rotatorios de la industria cementera pertenecen a los equipos industriales con las masas más grandes movidas. El cilindro ligeramente inclinado (con una longitud de más de $100 \mathrm{~m}$. y diámetros de hasta $8 \mathrm{~m}$.) se alimenta por un lado con la mezcla de la harina cruda. Del otro lado se encuentra el quemador, que produce temperaturas de hasta $1500^{\circ} \mathrm{C}$.

Por medio del proceso continuo de calcinación, la mezcla cruda se deshidrata y se compacta para formar piezas de "clinker" de tamaños irregulares. El "clinker" sale del horno, pasa por un enfriador y va al molino de cemento.

Hay dos procesos principales de calcinación: el húmedo y el seco. Cuando la harina cruda entra al horno mezclada con agua (esto lo facilita la 
etapa anterior del molino), se trata del proceso húmedo; cuando la mezcla cruda entra seca al horno, se trata del proceso seco. Como el proceso húmedo requiere mucha más energía para calcinar, es considerado técnicamente obsoleto.

Dos innovaciones caracterizan el desarrollo tecnológico del horno rotatorio reciente. Ambas innovaciones aumentan la capacidad instalada manteniendo constantes las dimensiones; por medio de una mejor utilización de la energía transformada en calor.

La primera innovación consiste en la introducción de intercambiadores de calor o precalentadores. Pasando por hasta 4 etapas, la mezcla cruda se calienta por medio de los gases calientes que salen del horno. Cuando sale de la última etapa del precalentador y entra al horno, ya tiene una temperatura entre $800^{\circ} \mathrm{C}$ y $1000^{\circ} \mathrm{C}$. El precalentador tiene una ventaja adicional ya que filtra los gases de escape. Gran parte de las partículas que contienen los gases se pueden recuperar con colectores de polvos y filtros y después reciclar al proceso. El intercambiador de calor es una invención de la compañía alemana Humboldt que llegó a su madurez comercial a principios de los años 50. Mientras tanto, los principales proveedores de equipo para la industria cementera ofrecen sus propios sistemas.

La segunda innovación importante es el sistema de precalcinación, desarrollado por productores japoneses de equipo cementero y comercializado por éstos a principios de los años 70 . Se precalcina la mezcla cruda en una cámara de combustión estacionaria entre precalentador y horno utilizando, por un lado, el calor que se produce en el enfriador del "clinker" y por el otro, una flama adicional. Comparado con sistemas anteriores, la introducción del precalcinador permite, por lo menos, duplicar la cantidad de material que pasa por el horno, y así aumentar significativamente la capacidad instalada sin ampliar las dimensiones del horno. El aumento de las dimensiones del horno lleva consigo una serie de problemas técnicos todavía no solucionados. Por ejemplo, el aumento de la longitud del-cilindro causa serios problemas de rodamiento y el aumento del diámetro, problemas del refractario.

El cuadro 2 resume algunas de las características de las dos innovaciones mencionadas. Los valores aproximados se refieren a las variaciones inducidas por la introducción de estas innovaciones.

En 1978, en las 28 plantas cementeras trabajaban un total de 68 hornos rotatorios. 9 de éstos tienen proceso húmedo y producen entre 200 tons. y 1000 tons, de "clinker" por día. Los 59 hornos restantes son de proceso seco y tienen capacidad diaria para la producción de "clinker" de 120 a 3000 tons. En muchas plantas se encuentran los equipos más modernos al lado del equipo que se adquirió en los años 40 y hasta antes todavía. La edad promedio de los hornos en 1978, era de aproximadamente 18 años y se verá reducida en 1983 a 15 años o menos. Los hornos que se instalaron a partir de la mitad de los años 60 cuentan generalmente con 
Cuadro 2

LAS dos Últimas INNOVACIONES MAYORES DEL PROCESO DE CALCINACIóN

\begin{tabular}{lccc}
\hline Caracteristicas & $\begin{array}{c}\text { Horno } \\
\text { tradicional } \\
\text { con enfriador }\end{array}$ & $\begin{array}{c}\text { Horno con } \\
\text { precalentador } \\
\text { y enfriador }\end{array}$ & $\begin{array}{c}\text { Horno con precalentador } \\
\text { precaloinador } \\
\text { y enfriador } \\
\text { mas grande }\end{array}$ \\
\hline $\begin{array}{l}\text { Año de introduccion } \\
\text { del-sistema }\end{array}$ & antes & 1950 & 1970 \\
$\begin{array}{l}\text { Capacidad instalada } \\
\text { (toneladas por dia) }\end{array}$ & 2400 & 2400 & -5000 \\
$\begin{array}{l}\text { Consumo de combusti- } \\
\text { ble (m3/Toneladas) }\end{array}$ & 200 & 95 & 100 \\
Longitud (m) & 200 & 80 & 80 \\
Dímetro (m) & 5 & 5.17 & 5.17 \\
\hline
\end{tabular}

FuENTE: Levantamientos propios.

intercambiadores de calor de hasta 4 etapas. Muchas veces, estos precalentadores fueron montados algunos años después de la instalación del horno. La mayoría de los nuevos proyectos que van a iniciarse en los próximos 3 años preven un sistema de precalcinación. Los primeros hornos con precalcinadores entrarán en operación a mediados de 1981.

Se observa una clara tendencia a instalar hornos más grandes. En los años 60 se instalaron pocos hornos con más de 1000 tons. de "clinker" diarios. En los años 70 la capacidad instalada promedio de los nuevos hornos era de alrededor de 1500 tons./día y actualmente es de alrededor de 2500 tons./día. Como un horno grande se opera con el mismo personal que un horno pequeño parecería que, por los problemas crecientes de mantenimiento se prohibirá la introducción de hornos aún más grandes. Debido a estas razones y por el alto costo de la inversión inicial actualmente los cementeros prefieren instalar en forma sucesiva dos hornos de 2500 tons. diarias en lugar de uno de 5000 tons. por día.

No sólo en el departamento de calcinación la industria cementera de México se encuentra al frente de la tecnología moderna a nivel internacional. Hoy en día las dos plantas que están en construcción también cuentan con una serie de elementos tecnológicos de alta sofisticación en otros departamentos. En una planta, por ejemplo, será instalado un sistema de trituración de tres etapas, considerado el más desarrollado para contrarrestar variaciones en la materia prima; en la otra instalará un sistema de envase automatizado - uno de los primeros en el mundoque carga automáticamente los sacos de cemento en camiones, es controlado por una computadora, y que reduce significativamente la fuerza de trabajo en este departamento. Otra planta cuenta con un centro de capacitación de personal especializado en computación de control de procesos; es el primer centro de este tipo a nivel mundial. 
En suma, se puede concluir que la industria mexicana del cemento, en cuanto a equipo, ha alcanzado el nivel tecnológico de los países industriales tradicionales y, en algunos casos, hasta ha sobrepasado a estos países y se encuentra al frente del desarrollo técnico de maquinaria a nivel mundial. Para lograr este nivel tecnológico, los cementeros mexicanos compran continuamente tecnología incorporada y no incorporada del exterior. Hoy, después de casi 80 años de producción nacional de cemento, todavía se importa gran parte de la maquinaria y se contrata a consultores técnicos extranjeros.

\section{LOS PROVEEDORES DE TECNOLOGÍA}

\subsection{Tecnología incorporada}

Los hornos instalados hasta y durante los años 50 fueron provistos por 10 proveedores aproximadamente en su mayoría de nacionalidad norteamericana. Desde entonces se ha dado un proceso de concentración del mercado de la tecnología incorporada en equipo y maquinaria para la industria cementera mexicana, que elimina a muchos de los proveedores tradicionales. Hoy existen prácticamente sólo 2 proveedores que dominan el abastecimiento de la industria mexicana del cemento: F. L. Smidth de Dinamarca y Polysius de la República Federal de Alemania.

La preferencia de los cementeros mexicanos por la tecnología incorporada europea es evidente, no sólo en cuanto a los hornos, sino también en los otros departamentos de la cementera. Los cementeros mencionan como razones principales por su preferencia, la calidad probada de la maquinaria europea y los servicios rápidos y efectivos de reparación y mantenimiento que prestan los proveedores europeos. Pero la razón más importante es, sin lugar a duda, el hecho que ambos proveedores mantienen oficinas propias bien equipadas en México que les permiten tener un contacto permanente con los clientes.

La oficina de F. L. Smidth en México se estableció en 1963 y cuenta ahora con 40 empleados, casi todos mexicanos. POLYSIUS tiene su propia oficina en México desde 1974 a través de la participación directa en una representación existente y actualmente tiene 16 empleados, en su gran mayoría mexicanos o alemanes nacionalizados.

F. L. Smidth es una empresa independiente que se dedica a la producción y comercialización de equipo para la producción de cemento y para la industria minera, y tiene talleres de fabricación en Dinamarca, Alemania, Estados Unidos, Brasil y oficinas de representación en muchos otros países. Polysius es una filial del gran consorcio industrial alemán Krupp; el equipo para producir cemento representa alrededor del $90 \%$ de las ventas totales; las otras especialidades son transportadores neumáticos, equipo para la industria siderúrgica y equipo de peletización. 
Las filiales más importantes, algunas también con fabricación, están en Francia, Inglaterra, España, África del Sur, Estados Unidos y Brasil.

Las principales funciones de las oficinas mexicanas proveedoras de equipo cementero, son el contacto con los clientes, el contacto con los suministradores mexicanos, la preparación y ejecución de proyectos -incluida una parte de ingeniería- y los servicios posteriores a la venta (refacciones y reparaciones). La oficina de Polysius recientemente inauguró un taller propio de fabricación (sobre todo de estructuras metálicas y pailería); manifestó que la capacidad existente de talleres de contratistas mexicanos no es suficiente.

Ambos proveedores ofrecen todo el equipo de una cementera aún sin producirlo ellos mismos. Muchas máquinas se reciben de otros productores y gran parte de su negocio consiste en la combinación de los elementos de tecnología incorporada, siendo contratistas generales. F. L. Smidth también ofrece la cementera llave en mano, y está actualmente montando una planta bajo este tipo de contrato. Sin embargo, por lo general en México, tienen que enfrentarse a una situación en la cual los clientes con sus consultores especifican detalladamente el tipo de máquinas a usarse y quiénes serán los productores. De esta manera, también existen varias plantas abastecidas de maquinaria por ambas firmas.

En cuanto a la penetración en el mercado mundial de equipo cementero, la participación de F. L. Smidth es de alrededor del $25 \%$ y la de Polysius de alrededor del $20 \%$, según la información suministrada por los mismos, tomando como base la capacidad instalada en 1979 e incluyendo las empresas licenciatarias. Los otros proveedores principales de equipo para la producción de cemento, que tienen menos del $10 \%$ del mercado mundial, son Humboldt (República Federal de Alemania), Gatx-Fuller (EE.UU.), Allis Chalmers (EE.UU.), Sket (República Democrática Alemana) y las tres empresas japonesas Mitsubishi, Ishikawajima Harima y Kawasaki Onada. A pesar de que todavía no existe єquipo japonés en México, pronto se espera el ingreso de tecnología japonesa a precios muy competitivos.

\subsection{Tecnología no incorporada}

Dado que no hay procesos de producción de cemento patentados, que la maquinaria es de fácil acceso en el mercado mundial y que la industria mexicana ya ha atraveșado por un largo período de acumulación de conocimientos, cabría suponer que no sería necesario contratar agentes extranjeros para el suministro de conocimientos técnicos. Pero sucede todo lo contrario. Cada año llegan más solicitudes de parte de las empresas mexicanas productoras de cemento a la Dirección de Inversiones Extranjeras y Transferencia de Tecnología de la Secretaría de Patrimonio y Fomento Industrial, pidiendo la inscripción de contratos sobre transferencia de tecnología no incorporada en el Registro Nacional de Transferencia de Tecno- 
logía, para poder deducir los costos de estos contratos de la cuenta de sus impuestos.

Hay varias razones para explicar este fenómeno, que dependen del tipo de tecnología objeto de la transferencia. Se pueden distinguir, según su contenido, dos grupos de contratos: uno se refiere a los nuevos proyectos, por ejemplo la ampliación de la planta con una nueva línea de producción. En este caso el contenido del contrato está bien especificado. Se trata generalmente de servicios de ingeniería básica y de detalle, de estudios técnicos y económicos de factibilidad, de la selección del equipo, de la capacitación del personal, y de la supervisión general del proyecto. Muchas veces los proveedores de este tipo de asistencia técnica tienen que cumplir garantías bien definidas para recibir la contraprestación. A la empresa cementera le conviene esta asistencia técnica porque los proyectos se llevan a cabo muy esporádicamente y carecen del personal especializado. Por lo tanto contratan un despacho de ingeniería o consultoría con experiencia en este campo.

El otro grupo de contratos es de tipo más general: asistencia técnica durante varios años para aumentar la productividad y reducir los costos de producción, incluyendo también elementos de ingeniería para pequeños proyectos. No se puede especificar tan concretamente el contenido de los servicios del proveedor y tampoco las metas a cumplir. Muchas veces el proveedor de este tipo de tecnología es el despacho de ingeniería del mismo grupo internacional al cual pertenece la compañía receptora a través de la participación en el capital social. Se corresponde con la lógica del funcionamiento de un grupo internacional suponer que de esta manera trata de alimentar sú despacho de ingeniería centralizado.

Sin embargo, hay que admitir que el equipo para producir cemento es cada vez más complejo, posee mayor capacidad y control automático de proceso, de tal manera que no es posible para los ingenieros de una cementera mantener actualizados sus conocimientos. A veces un consultor externo puede juzgar más objetivamente los problemas existentes en el aparato productivo y aumentar con pocas modificaciones el aprovechamiento de la capacidad instalada. Un caso clásico por el cual no se pueden concretar los servicios es, por ejemplo, la introducción de mantenimiento preventivo para toda la planta.

Los tres proveedores principales de tecnología no incorporada que operan en México, son: Holderbank Financiere Glaris (Suiza), Blue Circle Industries (Inglaterra) y Asland/Sereland (España). Mientras que el último es un grupo de consultores, los dos primeros son departamentos centralizados de ingeniería y consultoría de empresas trasnacionales productoras de cemento que venden en México sus servicios técnicos a las empresas miembro del grupo internacional. Holderbank tiene participación en el capital social de más de 50 cementeras en el mundo entero, mantiene un centro de entrenamiento de personal con una planta experimental en EE.UU. y el departamento de ingeniería y consultoría en 
Canadá y da respaldo financiero al grupo cementero más dinámico de México. Blue Círcle opera más de 150 hornos en diferentes países y maneja el centro de investigación tecnológica de cemento más grande del mundo.

En el desarrollo histórico de la tecnología cementera se observan varias etapas según los agentes que dominan y determinan la tecnología. En el siglo pasado los mismos cementeros desarrollaban su propia tecnología. A partir de fines de siglo pasado se inicia un proceso de separación entre los productores de cemento y los productores de los medios de producción para el cemento. Se forman empresas especializadas en equipo cementero que cada vez contribuyen en mayor grado a la internacionalización de la producción cementera en un proceso de autoconcentración.

A partir de los años 50 , con la creciente automatización de los procesos y con la introducción de los nuevos sistemas electrónicos de control, surge un nuevo agente tecnológico: el ingeniero consultor, como intermediario entre el productor de cemento y el productor de equipo.

El "ingeniero consultor" puede ser una empresa independiente, un departamento centralizado de una empresa productora de cemento o productora de equipo. El desarrollo de los últimos años indica que cada vez más, las empresas trasnacionales productoras de cemento son las que cumplen la función de consultoras de ingeniería. Hasta ahora no ha habido funciones entre productores de cemento y productores de equipo, lo cual sería una condición para la formación y la extensión del capital tecnológico. Únicamente en Japón el sector de maquinarias pesadas ha cooperado con el sector cementero en la búsqueda de innovaciones y éstas han sido desarrolladas en base a tecnología importada y esfuerzos comunes de investigación y desarrollo experimental.

El proceso básico de producción del cemento sigue siendo el mismo que el utilizado en los comienzos de la producción cementera a nivel industrial. Desde entonces todas las innovaciones se concentraron en el incremento de la productividad en este proceso. Muchos expertos del sector comparten la opinión respecto a que son escasas las posibilidades de un mayor desarrollo tecnológico de este proceso y que éste pronto llegará a sus propios límites, cuando las necesidades económicas exijan mayores avances en el aumento de la productividad.

\section{LAS FASES DE TRANSFERENCIA DE TECNOLOGÍA}

A continuación se estudiarán las etapas cronológicas del proceso de transferencia de tecnología. Se hará referencia a la instalación de una nueva línea de producción completa, ya sea a la ampliación de una planta existente o a la edificación de una nueva planta. En cada caso, las condiciones son muy diferentes y necesariamente se procederá a simplificar para 
lograr la comprensión general de la transferencia de tecnología en la industria cementera, enfocada como un proceso histórico. Así se pueden distinguir 5 fases principales:

1. Fase de decisión

2. Fase de obra civil

3. Fase de montaje

4. Fase de pruebas y ajustes

5. Fase de operaciones

Desde el momento de la firma de los contratos con los proveedores hasta el día del inicio de las operaciones, transcurren alrededor de dos años y medio para la erección de una nueva línea de producción o de una nueva planta con capacidad instalada de 2500 tons. de "clinker" diarias. Para la fase de decisión se calcula más o menos un año. La gráfica 2 muestra cómo las diferentes fases se superponen.

\section{Gráfica 2}

LAS FASES DE TRANSFERENCIA DE TECNOLOGÍA

\begin{tabular}{|c|c|c|c|c|}
\hline FASES & PRIMER AÑO & SEGUNDO AÑO & TERCER AÑO & CUARTO AÑO \\
\hline \multicolumn{5}{|l|}{ Decisión } \\
\hline \multicolumn{5}{|l|}{ obra civil } \\
\hline \multicolumn{5}{|c|}{ Montoje mecónico } \\
\hline \multicolumn{5}{|c|}{ Montoje eléctrico } \\
\hline \multicolumn{5}{|c|}{ Pruebos y ojustes } \\
\hline Operaciones & & & & \\
\hline
\end{tabular}

\subsection{Decisión}

En la fase de decisión se planean todos los elementos de la inversión total relacionada con la transferencia de tecnología. Se empieza con la recolección de información de revistas técnicas, material informativo de los proveedores de tecnología y visitas a otras plantas de cemento tanto locales como en el exterior. Es en esta fase cuando intervienen los despachos de ingeniería o consultoría y prestan sus servicios de ingeniería básica. Se lleva a cabo un estudio de mercado para determinar la capaci- 
dad instalada del nuevo proyecto. Se realizan estudios técnicos y económicos de factibilidad, vienen consejeros de los proveedores de tecnología y se hace una licitación o concurso con las especificaciones técnicas del proyecto. Entonces se integra una comisión formada por el director general de la empresa, el director técnico y el director financiero.

Hay múltiples formas contractuales. En un caso extremo se opta por un paquete tecnológico "llave en mano", en el cual el proveedor de tecnología es contratista general y abastece la línea de producción completa desde la trituración hasta el envase. En la industria mexicana del cemento estos contratos "llave en mano" son raros. En el otro extremo y cuando existe capacidad propia de personal, el departamento técnico de la cementera combina los componentes técnicos según criterios propios y se contrata directamente a los proveedores de los diferentes equipos. En la mayoría de los casos existe un contratista general para el equipo principal y se celebran contratos individuales para los equipos adicionales.

El criterio más importante para la decisión es la calidad del equipo; experiencia en el pasado y cooperación anterior con el proveedor son factores que juegan un papel importante. Los costos de operación, sobre todo para el personal necesario y el consumo de energía, pueden también influir fuertemente en la decisión. Sin embargo, muchas veces ciertos aspectos financieros son más importantes que el aspecto técnico, sobre todo cuando se financia con créditos extranjeros, que obligan a comprar la tecnología en el país de origen del crédito o cuando un proveedor otorga mejores facilidades de crédito o tipos de pago que el otro. En la medida en que la tecnología se estandariza y los precios se acercan, los criterios financieros determinan cada vez más la decisión.

\subsection{Obra civil}

Poco después de firmar los contratos empiezan los trabajos de la obra civil. Por un lado, se trata de obras de infraestructura (caminos, conexiones ferroviarias, subestaciones eléctricas, etc.), y por el otro, de los edificies para la maquinaria y para las oficinas. Muchas veces, en el caso de una nueva planta, parte integral del proyecto es la construcción de viviendas donde habitará el nuevo personal. Son exclusivamente constructoras mexicanas, especializadas en construcciones industriales, quienes realizan la obra civil. Para algunas partes de la obra civil se necesitan conocimientos especiales, experiencia y tecnología sofisticada; por ejemplo, en el caso de los cimientos que cargan las máquinas más pesadas y los silos gigantescos (hasta $80 \mathrm{~m}$. de altura y $25 \mathrm{~m}$. de diámetro), para la construcción de los cuales se utiliza el sistema de "cimbra deslizante".

El personal para llevar a cabo la obra civil varía según el avance de la obra. En el pico de la construcción trabajan hasta 1000 personas en la obra, más o menos la mitad de ellos como peones que las constructoras contratan en la región. Se calculan entre 1200 y 1400 hombres/año para la obra civil de una nueva planta con capacidad instalada de 2500 tone- 
ladas de "clinker" diarias, sin contar las construcciones adicionales de oficina y vivienda. Por lo general, poco personal de la cementera participa en la obra civil, sólo algunos ingenieros que supervisan si las construcciones corresponden a las exigencias de los planos y si la obra avanza según las fechas establecidas en el plan general del proyecto.

\subsection{Montaje}

En la fase del montaje se instalan los equipos mecánicos y eléctricos. Una parte del equipo es importada, la otra se fabrica en talleres nacionales y una tercera se hace en el lugar de la obra. Se empieza con el montaje mecánico y a partir de cierto nivel de avance entra el montaje eléctrico y la instalación de los instrumentos. Aparte de las herramientas comunes de mecánicos y electricistas, se requiere cierto equipo especial, por ejemplo, grúas para el manejo de las partes pesadas.

El origen del personal de montaje varía mucho según el tipo de contratación. Cuando se trata de un contratista general, él manda un equipo de técnicos para supervisar el montaje durante todo el período. Cuando existen varios contratos individuales con los proveedores de la tecnología, cada uno de ellos se responsabiliza de la instalación de su equipo a través de la supervisión de por lo menos un técnico, durante el tiempo que insuma el montaje.

En proyectos grandes se contratan compañías especializadas en montaje industrial para llevar a cabo el montaje. Para el montaje mecánico de una nueva planta con capacidad diaria de 2500 tons. de "clinker", se necesitan entre 150 y 200 personas durante todo el tiempo del montaje. Un $40 \%$ de ellos requiere calificación especial. En total se calcula, para un proyecto de este tamaño, alrededor de 300 hombres/año, únicamente para el montaje mecánico. El personal de la empresa cementera puede participar en esta fase de montaje en dos niveles: a nivel de los trabajadores, cuando tiene suficiente personal en el caso de proyectos menores, y a nivel de supervisión y de control a través de los ingenieros del departamento técnico de la empresa.

Según la participación de personal propio en la fase de montaje se distinguen dos estrategias opuestas, conocidas en el medio como el "sistema americano" y el "sistema europeo". Ambos sistemas por separado y sistemas mezclados pueden hallarse en la industria mexicana del cemento. El "sistema americano" rígido prevé, que el personal propio preparado mediante cursos de capacitación, se incorpore cuando la instalación de la nueva planta está terminada para ponerla en operación. La instalación la llevan a cabo compañías externas, también a nivel de supervisión y control. El "sistema europeo" rígido trata de involucrar lo máximo posible al personal propio de la empresa en la fase de montaje, no sólo a nivel de supervisión y control, sino también a nivel de trabajadores, sobre todo a aquellos que después se encargarán del mantenimiento; de esta manera 
se espera que el personal de la nueva planta aprenda más directamente los detalles técnicos del funcionamiento del equipo. Un sistema mezclado, frecuentemente adoptado por la industria mexicana del cemento, consiste en la participación de algunas personas de la cementera en la fase de montaje que después tomarán puestos directivos, y que serán auxiliados por ingenieros de proyecto externos con experiencia en este campo, contratados para funciones de supervisión y control del montaje.

\subsection{Pruebas y ajustes}

En la fase de pruebas, se pone en marcha el conjunto de máquinas después de efectuar una serie de adaptaciones, modificaciones y afinaciones de todos los componentes para llegar a la capacidad instalada nominal. Cuando se termina el montaje, primero se hace la prueba de marcha en frío de todas las máquinas en forma individual. Después empiezan las pruebas de rendimiento para cumplir las garantías establecidas en los contratos. Estas garantías se refieren en primer lugar, a un volumen determinado de producción en un determinado tiempo y de una calidad bien definida, al consumo máximo de medios auxiliares para la producción (energía eléctrica y calorífica) y a otros criterios de rendimiento (por ejemplo, temperatura del "clinker" y emisión de polvo).

Como la tecnología de procesos en la producción de cemento es suficientemente conocida, se calcula un período de aproximadamente una semana para las pruebas de rendimiento. Las pruebas terminan con la puesta en marcha de la nueva línea de producción o de la nueva planta y la toma de mando. Para poder entregar el nuevo equipo al cliente, los proveedores tienen que cumplir con las garantías especificadas en los contratos, por ejemplo, que durante 72 horas se aproveche la capacidad instalada nominal, de la calidad requerida del cemento, sin sobrepasar ciertos valores de consumo.

Si las partes contractuales se muestran satisfechas con los resultados de la prueba de la toma de mando, se ponen en marcha las operaciones, y se retiran los técnicos de los proveedores. Si no, tienen que buscar y arreglar las fallas, pagando multas de convención, hasta que tengan los resultados garantizados. El personal de la empresa que se encarga de las operaciones y que ha sido capacitado previamente dentro de la misma, pasa durante un cierto tiempo (se consideran por lo menos 6 meses) por una fase de ajustes en la cual aprende los detalles del nuevo equipo para optimizar su funcionamiento (ver gráfica 3 ). Mientras que la fase de pruebas está bajo la tutela de los técnicos de los proveedores de tecnología, es el personal propio de operaciones el que realiza los ajustes. Hay varios casos en la industria mexicana del cemento que muestran un aprovechamiento de la capacidad instalada de más del $100 \%$, mediante una política deliberada de optimización de los diversos componentes del equipo. Aunque la tecnología de proceso es bastante conocida, cada nueva unidad de producción 
requiere su propia atención en cuanto a la curva de aprendizaje. Así la fase de pruebas y ajustes juega un papel decisivo en la asimilación de la tecnología transferida.

Gráfica 3

CURVA DE APRENDIZAJE

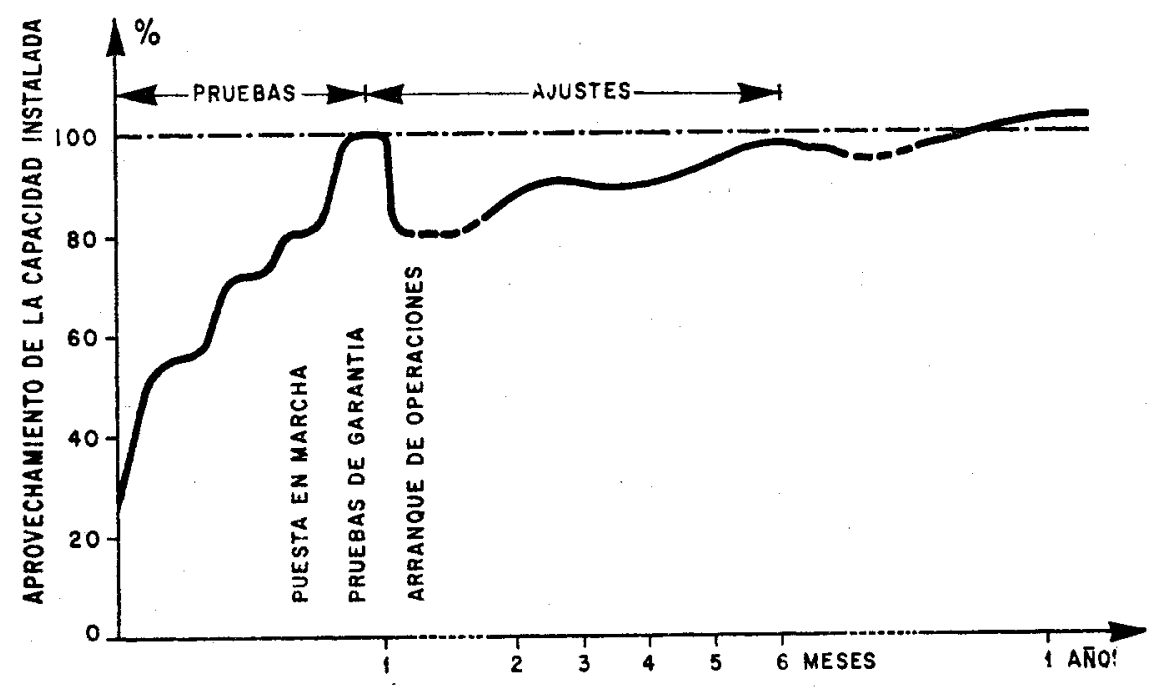

\subsection{Operación}

La fase de operaciones pertenece todavía al proceso de transferencia de tecnología, ya que en esta fase se dan los resultados y se presentan los problemas involucrados en la tecnología transferida. Si en la fase de pruebas y ajustes se habló de la asimilación de la tecnología transferida por parte del personal propio de la cementera, se puede caracterizar la fase de operaciones como un proceso de apropiación definitiva de la tecnología transferida. Particularmente son dos los tipos de trabajo que permiten captar la capacidad tecnológica propia de la empresa receptora de tecnología: los trabajos regulares de mantenimiento y los trabajos imprevisibles de reparación. En cuanto a los últimos se pueden distinguir tres tipos, según la gravedad de la falla y las posibilidades de arreglarla con medios propios. En el primer caso, el departamento técnico y el taller de mantenimiento de la empresa pueden reparar la falla sin ninguna intervención de afuera y con las refacciones del propio almacén. En el segundo caso, se necesita la ayuda de un taller metalmecánico externo o de la representación na- 
cional del proveedor de la tecnología, y finalmente hay reparaciones tan difíciles que exigen la intervención de especialistas de la sede matriz del proveedor y hasta la importación urgente de partes de la maquinaria traídas en avión.

Todos los técnicos del sector están de acuerdo en que el problema más difícil y costoso de mantenimiento es el del refractario: el recubrimiento de tabiques especiales en el interior del horno que protege al cilindro de acero de deformaciones. Debido a la operación continua a altas temperaturas y a la carga mecánica permanente, algunos tabiques refractarios se desatan con el tiempo de su conjunto. Existe el peligro de que en esta parte el cilindro de acero del horno se deforme por el calor. Se calculan en general, de 2 hasta 3 paros anuales para cambiar partes del refractario. Estos trabajos duran de 1 hasta 2 semanas y causan altos costos directos y ganancias perdidas. Las estrategias de las diferentes cementeras para resolver este problema de mantenimiento varían. En algunas plantas se para el horno solamente cuando es absolutamente necesario para un mantenimiento correctivo. Otras plantas adoptan un sistema de mantenimiento preventivo en base a series estadísticas y paros probables. Este último sistema se puede considerar como un indicador de una gran capacidad tecnológica propia, porque se transforman de una manera productiva los conocimientos sobre el horno individual y sobre las características de los múltiples tabiques refractarios en el mercado.

La actitud de los receptores de tecnología hacia el proceso de transferencia varía en todas las fases entre recepción pasiva y participación activa. El tamaño de la planta o el ser miembro de un grupo cementero influye menos en esta actitud que la política deliberada de la empresa en materia tecnológica, que se manifiesta, por ejemplo, en la forma de preparar y capacitar al propio personal. Ser afiliado a un grupo cementero trasnacional tampoco garantiza la apropiación activa de la tecnología importada; sin embargo, las plantas correspondientes cuentan muchas veces con la asistencia técnica de un equipo de ingenieros, prestados por la matriz durante todo el período de la realización del proyecto, que puede redundar en menos fallas y ahorro de tiempo y costos.

Los problemas de apropiación de la tecnología transferida se presentan menos a nivel ingenieril que a nivel operativo y se articulan en la fase de operaciones en la incapacidad del personal técnico de reaccionar rápido y efectivamente a nuevas situaciones, y en las áreas de reparaciones y mantenimiento. Así la dependencia del exterior a nivel de la empresa no solamente se perpetúa a través de la necesidad de importar refacciones, sino también respecto a los conocimientos técnicos necesarios para mantener el funcionamiento del aparato productivo bajo condiciones cambiantes. En la producción de cemento, tan intensiva en el uso de capital, cada operador tiene bajo su responsabilidad un sistema demasiado complejo de funciones, y cada vez más complejo cuando se automatizan más los pro- 
cesos, de tal manera que la dominación de la tecnología dependen en gran medida de la preparación, capacitación y motivación de los trabajadores.

Otro factor, posiblemente el más importante, que convierte la función potencialmente emancipadora de la transferencia de tecnología en una represiva, es la estrategia seguida por los proveedores de tecnología de introducir una creciente sofisticación en sus equipos, obedeciendo a las leyes de valorización establecidas por los grandes grupos internacionales de cemento. Con el incremento de la automatización de los procesos y el control computarizado, se aumenta la necesidad de los cementeros de recurrir a la asistencia técnica de los consultores internacionales en campos cada vez más estratégicos de la producción. Por eso, lo que a primera vista se presenta como un nivel tecnológico de punta, no es más que el resultado de una dominación de pocos centros de control sobre el resto del mundo, mediante la transferencia de tecnología incorporada y no incorporada, dominación que se encubre tras la necesidad de desarrollo de las fuerzas productivas en forma de desarrollo tecnológico.

\section{Costo, peso y Contenido de importación de la tecnología}

\subsection{Inversión total}

Los datos presentados en este capítulo se refieren a dos nuevas plantas de cemento actualmente en construcción. Aunque los dos proyectos se distinguen bastante, tanto en lo técnico como en lo financiero, tienen características comunes que nos permiten hacer algunas aclaraciones respecto a la tecnología: ambas plantas pertenecen a grupos cementeros con participación extranjera en el capital social. La construcción de las dos plantas se inició en 1979 y la puesta en marcha se hará a mediados de 1981; tienen una capacidad instalada diaria de "clinker" de 2500 toneladas y una capacidad instalada anual de cemento de 900000 toneladas. Los datos principales sobre la inversión total están resumidos en el cuadro 3 .

Mientras que en el proyecto II la maquinaria proviene de diferentes proveedores y el montaje está coordinado por un equipo de ingenieros del grupo, en el proyecto I viene en un paquete tecnológico con un contrato "llave en mano", una de las razones del mayor costo de inversión. Sin embargo, hay que aclarar que parte del equipo del proyecto I está sobredimensionada con vistas a una ampliación en el futuro próximo. El grupo cementero se decidió por un paquete tecnológico, en este caso, debido a dos motivos importantes: no tiene suficiente personal propio de coordinación y supervisión, especialmente porque se están llevando a cabo ampliaciones en otras plantas simultáneamente, y quiere terminar el proyecto en el menor tiempo posible. A pesar de las diferencias en costo, se observa que las proporciones de los grandes grupos de costos son muy parecidas. 
Para la obra civil tenemos el $27.8 \%$ en el proyecto I y el $29.6 \%$ en el proyecto II, y para la maquinaria, inclusive montaje, el $50.0 \%$ en el proyecto I y el $50.9 \%$ en el proyecto II.

La intensidad en el uso de capital muy elevada en la producción de cemento se refleja en la alta inversión por puesto de trabajo nuevo: $\$ 10.9$ millones y $\$ 9.3$ millones respectivamente. Ambas plantas tienen una ad-

\section{Cuadro 3}

Comparación de dos nUevas plantas de CEMENTo

\begin{tabular}{|c|c|c|c|c|c|c|c|}
\hline & \multicolumn{4}{|c|}{ Froyecto $\mathrm{r}$} & \multicolumn{3}{|c|}{ Proyecto II } \\
\hline & \multicolumn{3}{|c|}{$\begin{array}{l}\text { Millones } \\
\text { de pesos }\end{array}$} & Porciento & \multicolumn{2}{|l|}{$\begin{array}{l}\text { Millones } \\
\text { de pesos }\end{array}$} & Porciento \\
\hline Obra Civil & $I$ & 000 & & 27.8 & 746 & & 29.6 \\
\hline Equipo mecanico & & & & & 886 & & 35.1 \\
\hline Equipo electrico & & & & & 202 & & 8.0 \\
\hline Montaje mecánico & & 800 & & 50.0 & 132 & & 5.2 \\
\hline Montaje eléctrico & & & & & 65 & & 2.6 \\
\hline Resto (1) & & 800 & & 22.2 & 490 & & 19.4 \\
\hline Inversion total & 3 & 600 & & 100.0 & 2521 & & 100.0 \\
\hline Paquete tecnol6gico & \multicolumn{4}{|c|}{ s1 } & \multicolumn{3}{|c|}{ si } \\
\hline $\begin{array}{l}\text { Capacidad inst. anual } \\
\text { (tons. de cemento) }\end{array}$ & \multicolumn{4}{|c|}{900000} & \multicolumn{3}{|c|}{900000} \\
\hline $\begin{array}{l}\text { Inversion total por } \\
\text { ton. de cemento (pesos) }\end{array}$ & \multicolumn{4}{|c|}{4000} & \multicolumn{3}{|c|}{2801} \\
\hline $\begin{array}{l}\text { Nuevos puestos de } \\
\text { trabajo }\end{array}$ & \multicolumn{4}{|r|}{330} & \multicolumn{3}{|r|}{270} \\
\hline $\begin{array}{l}\text { Inversion total por } \\
\text { puesto de trabajo (pesos) }\end{array}$ & \multicolumn{3}{|c|}{10909} & 091 & \multicolumn{3}{|c|}{9337037} \\
\hline
\end{tabular}

1 El resto de la inversión se refiere a impuestos de importación, seguros, fletes, capacitación de personal, etcétera.

FUENTE: Levantamientos propios.

ministración central en la Ciudad de México, de tal manera que la creación de empleos total se puede considerar ligeramente más alta. Parte del ahorro en personal en el proyecto II se explica por la existencia de más funciones centralizadas en la sede matriz y por un nuevo sistema de envase automatizado. La inversión por tonelada anual de cemento es de $\$ 4.000$ y $\$ 2.801$ respectivamente; se sitúan en los extremos de un promedio de $\$ 3.500$, que es la valoración actual del costo de inversión de una nueva línea de producción. 


\subsection{Equipo}

En el equipo que cuesta más o menos la mitad de los dos proyectos estudiados, incluyendo el montaje, la tecnología está incorporada. El cuadro 4 demuestra cómo se reparten los costos del equipo en los 6 departamentos, mencionados en el capítulo 3.

En el proyecto I se destaca la proporción de los departamentos de trituración y homogeneización. Se optó por la tecnología más sofisticada a nivel mundial, principalmente por dos razones: por un lado, la planta tiene que enfrentarse con el problema de una gran variación de la materia prima y, por el otro, quiere reducir al máximo el trabajo de la molienda de crudo con un sistema de trituración en tres etapas. En el proyecto II

\section{Cuadro 4}

DisTRIBUCIÓN DE LOS COSTOS DEL EQUIPO SEGÚN DEPARTAMENTOS TÉCNICOS (EN PORCIENTO)

\begin{tabular}{lcc}
\hline Departamentos técnicos & Proyecto I & Proyecto II \\
\hline Trituración & 16.3 & 12.8 \\
Homogenizacion & 12.3 & 8.3 \\
Molienda de crudo & 17.4 & 20.0 \\
Calcinacion & 25.4 & 25.5 \\
Molienda de cemento & 22.3 & 21.4 \\
Envase & 6.3 & 12.0 \\
Total & 100.0 & 100.0 \\
\hline
\end{tabular}

Nota: La información original contiene una parte de equipo que no está directamente relacionada con los seis departamentos (central de control, equipo móvil, equipo auxiliar, etc.); en el proyecto I esta parte representa el $14.0 \%$, en el proyecto II el $28.5 \%$ del total. Para homogeneizar los datos, se repartieron en partes iguales entre los seis departamentos.

FUENTE: Levantamientos propios.

se trabajará con una trituradora integrada de una etapa. En los departamentos de molienda de crudo y de cemento también hay una diferencia importante. Mientras que el proyecto II tiene un molino grande en cada departamento, en el proyecto I están instalando dos molinos menores, primero para tener menos problemas de mantenimiento y más versatilidad; en segundo lugar será suficiente la instalación de un tercer molino de igual tamaño en cada departamento para la ampliación futura con un horno adicional de igual capacidad. Estas diferencias en los departamentos de molienda son aún más notables porque en ambos casos se trata del 
mismo proveedor que también abastece al sistema de calcinación a las dos plantas.

Los sistemas de calcinación son bastante semejantes en ambos proyectos; representan un poco más de la cuarta parte del costo total del equipo: un precalentador de 4 etapas, sistema de precalcinación con ducto terciario, horno rotatorio con un diámetro del cilindro de $4.15 \mathrm{~m}$. y una longitud de $85 \mathrm{~m}$. y enfriador de "clinker" de parrilla. Otra diferencia que se observa en las proporciones correspondientes, se encuentra en los departamentos de envase: ambas plantas cuentan con envasadoras rotatorias de 8 boquillas, el proyecto I con 3 , el proyecto II con 2; además se introducirá en el proyecto II un sistema automático de alimentación de sacos y de carga en camiones, uno de los primeros en el mundo. La razón es ahorrar mano de obra; el departamento de envase, el departamento tradicionalmente más intensivo en el uso de la mano de obra de una cementera, sin esta automatización necesitaría 40 personas para operar.

El peso total del equipo de una planta cementera de este tamaño fluctúa entre 10000 y 12000 toneladas; es decir, se requieren entre 4 y 4.8 toneladas de equipo por cada tonelada de "clinker" producida por día. Más o menos un $15 \%$ corresponde a equipo eléctrico, y un $10 \%$ a equipo móvil. El equipo montado cuesta actualmente alrededor de $\$ 150000$ por tonelada, aproximadamente $\$ 140000$ para el equipo mecánico y $\$ 180000$ para el eléctrico. Los costos promedio de equipo sin montaje son de $\$ 120000$ y de $\$ 135000$ por tonelada, respectivamente.

\subsection{Contenido de importación}

Según información de las direcciones técnicas de las empresas cementeras, el contenido de importación en el valor total del equipo es de un $50 \%$. Los proveedores de tecnología confirman este dato, pero no querían especificarlo según departamentos o componentes, debido a que están manejando esta información únicamente para fines internos. Sin embargo, se consiguió información sobre la participación mexicana en la fabricación de equipo mecánico, tomando como base el peso de los principales componentes que en caso concreto representan el $94.9 \%$ del peso total del equipo mecánico; esta información, con una especificación por tipo de equipo y la participación nacional en su producción, se da a conocer en el cuadro 5.

Tomando como criterio la participación nacional en la fabricación, el equipo mecánico se puede clasificar en tres grupos: un grupo con equipo completamente fabricado en México, un grupo cuyo equipo se fabrica; en parte en México y en parte en el exterior y un grupo de equipo enteramente importado. El contenido de importación del peso de todo el equipo que se calcula en $43.7 \%$ significa, en comparación con el contenido de importación del valor, que se importa equipo con menos peso y/o mayor valor que el equipo nacional. Esto se verifica comparando el tipo de equipo 
de los tres grupos. En el primer grupo de equipo de fabricación completamente nacional se trata, principalmente, de equipo estandarizado simple, de uso general también en otros sectores industriales y con una alta proporción de estructura metálica y de pailería, componentes que tienen relativamente poco valor agregado por tonelada:

\section{Cuadro 5}

Fabricación NaCIONAL DE EQUIPo

\begin{tabular}{|c|c|c|c|}
\hline \multirow[b]{2}{*}{ Tipo de equipo } & \multirow[b]{2}{*}{$\begin{array}{c}\text { Peso } \\
\text { (toneladus) }\end{array}$} & \multicolumn{2}{|c|}{ Fabricación nacional } \\
\hline & & $\begin{array}{l}\text { Porciento } \\
\text { estimado }\end{array}$ & $\begin{array}{c}\text { Peso } \\
\text { (toneladas) }\end{array}$ \\
\hline I. Equipo totalmente nacional & & & \\
\hline $\begin{array}{l}\text { Ductos } \\
\text { Bandas transportadoras } \\
\text { Precalentador } \\
\text { Alimentadores de banda } \\
\text { Colectores de polvo } \\
\text { Torre de enfriamiento } \\
\text { Elevadores de cangilones } \\
\text { Ventiladores }\end{array}$ & $\begin{array}{r}750 \\
721 \\
273 \\
232 \\
96 \\
83 \\
80 \\
67\end{array}$ & $\begin{array}{l}100 \\
100 \\
100 \\
100 \\
100 \\
100 \\
100 \\
100\end{array}$ & $\begin{array}{r}750 \\
721 \\
273 \\
132 \\
96 \\
83 \\
80 \\
67\end{array}$ \\
\hline Subtotal & 2202 & 100 & 2202 \\
\hline II. Equipo parcialmente nacional & & & \\
\hline $\begin{array}{l}\text { Equipos afversos } \\
\text { Electrofiltros } \\
\text { Horno con enfriador } \\
\text { Transportadores especiales } \\
\text { Apilador } \\
\text { Alimentadores de zapato } \\
\text { Motores }\end{array}$ & $\begin{array}{r}1000 \\
735 \\
660 \\
166 \\
131 \\
110 \\
109\end{array}$ & $\begin{array}{l}70 \\
30 \\
70 \\
40 \\
30 \\
20 \\
70\end{array}$ & $\begin{array}{r}700.0 \\
220.5 \\
462.0 \\
66.4 \\
91.0 \\
22.0 \\
76.3\end{array}$ \\
\hline Subtotal & 2.911 & 56.3 & 1.638 .2 \\
\hline III. Equipo extranjero & $\cdot$ & & \\
\hline $\begin{array}{l}\text { Bolas de molino } \\
\text { Molinos } \\
\text { Recubrimiento interno de molinos } \\
\text { Quebradoras } \\
\text { Separadores } \\
\text { Cadenas de arrastre } \\
\text { Reductores de velocidad }\end{array}$ & $\begin{array}{r}710 \\
495 \\
258 \\
.156 \\
116 \\
95 \\
81\end{array}$ & $\begin{array}{l}0 \\
0 \\
0 \\
0 \\
0 \\
0 \\
0\end{array}$ & $\begin{array}{l}0 \\
0 \\
0 \\
0 \\
0 \\
0 \\
0\end{array}$ \\
\hline Subtotal & 1911 & 0 & 0 \\
\hline Total & 7024 & 54.7 & 3. 840.2 \\
\hline
\end{tabular}

FUENTE: Levantamientos propios.

En el segundo grupo se encuentra equipo más especializado, más complicado y de uso menos general que en el primer grupo; otra vez la fabricación nacional se concentra en elementos metálicos de soporte estructural y de pailería. También los motores menores (hasta de 400 caballos) se producen en el país. Las partes de importación tienen características especiales que no se consiguen en México. Son las láminas y los alambres de aleaciones especiales de los electrofiltros y los motores grandes, por ejemplo.

El caso del horno merece una atención especial. Todos los hornos que 
se instalan en México necesitan importar las llantas y las virolas debido a limitaciones técnicas de los talleres nacionales. Lo mismo pasa con la corona del horno, que por un lado necesita fundición en una colada, y por el otro un torno especial para cortar los dientes; sólo pocos talleres en el mundo tienen las posibilidades técnicas para producir engranes de este tamaño gigantesco. En el caso estudiado se importa también la lámina del cilindro, la cual se rola en un taller nacional. En otros casos se manda a rolar la lámina en el exterior, porque los planos técnicos prevén secciones tan anchas que sobrepasan la capacidad nacional para ahorrar en soldaduras de obra, sobre todo en la sección crítica de la temperatura más alta de calcinación.

El tercer grupo, que se importa enteramente, comprende equipo muy especializado que no se puede desintegrar y de materiales con calidades muy especiales. Esto se refiere, por ejemplo, a los molinos, sus bolas y su recubrimiento interno de alta duración de vida de los cuales depende en gran medida el tiempo de operación sin mantenimiento.

Resumiendo se puede decir que la fabricación nacional depende del grado de especialización del equipo, de materiales especiales que no se consiguen en el mercado nacional, y del equipo especial necesario que no se encuentra en los talleres nacionales. Otro factor es la capacidad cuantitativa de los talleres nacionales; en los proyectos estudiados se calcula que hasta el $75 \%$ del valor del equipo (en vez del $50 \%$ ) podría técnicamente producirse en el país, de no existir tantos cuellos de botella en la capacidad de los pocos talleres nacionales, debido al auge general en el sector industrial.

Hasta hace pocos años, los proveedores principales y las empresas cementeras preferían el equipo importado al nacional, no tanto, por la calidad que en ambos casos definía y controlaba el mismo proveedor de tecnología, sino por el menor costo del equipo importado. Hoy, las empresas cementeras insisten en la máxima participación nacional en la fabricación del equipo para poder aprovechar los incentivos fiscales. Por ejemplo, se otorga, bajo ciertas condiciones, una exención de impuestos de un $20 \%$, cuando la participación nacional en el valor de equipo es de más de un 50 por ciento.

Cabe señalar que los proveedores de tecnología también se interesan en reducir cada vez más el contenido de importación del equipo que ofrecen. Como ya realizan gran parte de su negocio, no con la producción de su equipo, sino con la ingeniería y la comercialización, les conviene nacionalizar su producción al máximo para reducir, en particular, los costos de transporte y de mano de obra. De esta manera, el proceso de internacionalización de la producción de cemento y de la tecnología cementera, induce lógicamente a un proceso de nacionalización aprovechando las condiciones diferenciales de producción e igualándolas al mismo tiempo a nivel mundial. 


\section{CONCLUSIONES}

1) El desarrollo de la industria mexicana del cemento, que comenzó a principios del siglo, está acompañado por una continua transferencia de tecnología y cuenta actualmente con las instalaciones más modernas y la tecnología más avanzada a nivel mundial. Se sigue importando la tecnología en dos formas: la tecnología incorporada en maquinaria y equipo a través de la contratación de proveedores extranjeros, y la tecnología no incorporada a través de contratos de asistencia técnica.

2) La expansión rápida de la industria se combina con un proceso de concentración de los productores de cemento que lleva a la dominación del mercado nacional por pocos grupos. Paralelamente observamos una reducción de los proveedores de la tecnología, los cuales determinan cada vez más las condiciones de la producción de cemento a nivel internacional.

3) A pesar de que los procesos de producción del cemento son bien conocidos, la mayor parte de los productores nacionales celebra contratos de asistencia técnica y suministro de conocimientos técnicos con el extranjero. Estos contratos se refieren a nuevos proyectos o a asistencia técnica durante un determinado período y ayudan a los cementeros a actualizar sus conocimientos sobre la creciente sofisticación del aparato productivo.

4) A nivel nacional, la dependencia tecnológica se perpetua mediante la importación permanente de tecnología extranjera. A nivel de empresa se desarrollan estrategias para relativizar la dependencia tecnológica. Para una asimilación y apropiación de la tecnología transferida son indispensables, por ejemplo, la preparación del personal, la participación del personal propio en las diferentes fases del proceso de transferencia y la atención que se presta a la curva de aprendizaje del nuevo equipo instalado.

5) Los problemas del funcionamiento de la tecnología transferida se presentan en la fase de las operaciones y más a nivel operativo que a nivel ingenieril. Como el desarrollo tecnológico está caracterizado por una creciente automatización y complejidad del equipo, los operadores tienen cada vez mayores responsabilidades. Por eso, el funcionamiento de la tecnología depende, en última instancia, de los trabajadores, de su capacitación y motivación.

6) En cuanto a la tecnología incorporada podemos constatar una creciente participación de la fabricación nacional. Actualmente, el contenido de importación en el valor total del equipo es, aproximadamente, de un $50 \%$. El contenido de importación en el peso es inferior, debido a que se importa equipo con menos peso y/o mayor valor que el equipo nacional. La fabricación nacional de equipo está todavía limitada, cuantitativamente por la baja capacidad de los talleres nacionales, y cualitativamente por el grado de especialización tanto del equipo, como 
de los materiales requeridos y de los medios de producción necesarios.

7) Los productores de cemento al igual que los proveedores de la tecnología incorporada están interesados en una mayor integración nacional de la fabricación del equipo: los primeros, para aprovechar los estímulos fiscales correspondientes, y los últimos, para reducir los costos de producción, en particular, los costos de transporte y de mano de obra.

8) El proceso de internacionalización de la producción de cemento, llevado a cabo y controlado por pocos agentes a través de la transferencia de tecnología, induce un proceso de nacionalización, no sólo de la producción de cemento, sino también de la tecnología. Este proceso aprovecha las condiciones diferentes de producción y tiende a generalizarlas, con el tiempo, a escala mundial.

9) El caso de la transferencia de tecnología en la industria mexicana del cemento indica que una nueva fracción de capital está en formación: el capital tecnológico. Se independiza y autonomiza como parte del capital industrial a través del proceso de internacionalización y crea sus propios agentes y sus propias leyes de valorización. El capital tecnológico, producto de la internacionalización del capital, se establece como el nuevo motor de este proceso, al lado del capital financiero. 